Short Report

\section{Problems in the therapy of mixed malarial infections: a case of infection with Plasmodium falciparum and $P$. malariae treated with mefloquine and halofantrine}

\section{F. J. Hess, H. D. A. Kilian, H. D. Nothdurft and T. Löscher Department of Tropical Medicine and Infectious Diseases, University of Munich, Munich,} Germany

Mixed malarial infections of Plasmodium falciparum and $P$. malariae are not uncommon but they may often be overlooked because there is a tendency for $P$. falciparum to predominate and the parasitaemia with $P$. malariae is usually very low.

Therapy of cases of mixed infections with $P$. falciparum and $P$. malariae, $P$. vivax or $P$. ovale should clearly be aimed at $P$. falciparum as the potentially lethal infectious agent (COOK, 1988), followed by a course of primaquine in the case of $P$. vivax or $P$. ovale. For non-immune subjects, mefloquine and halofantrine are the currently recommended first line drugs for uncomplicated falciparum malaria imported from African countries where chloroquine resistance is common (WHO, 1992).

However, very limited information is available concerning the efficacy of these 2 drugs in the treatment of quartan malaria. DIXON and co-workers (1983) have successfully used mefloquine to treat a case of $P$. malariae and they reported rather slow resolution of fever and parasitaemia. Similarly, WEINKE et al. (1993) reported the successful treatment of 2 cases of $P$. malariae infection (one mixed with $P$. falciparum) with halofantrine, but in both reports the follow-up period was only $28 \mathrm{~d}$.

Here we report a patient with a mixed infection of $P$. falciparum and $P$. malariae treated initially with mefloquine and subsequently with halofantrine; the possible failure of both drugs is discussed.

In July 1991 a 46 years old German woman presented at a peripheral hospital in Germany with fever $\left(39.2^{\circ} \mathrm{C}\right)$, chills, headache and anorexia of $3 \mathrm{~d}$ duration, $12 \mathrm{~d}$ after returning from 2 weeks' holiday in Kenya. She denied having taken any malaria chemoprophylaxis or anti-malarial treatment. The diagnosis of falciparum malaria was made by examination of blood films which had been sent to our institute for confirmation. The initial parasitaemia was $3 \%(136000 / \mu \mathrm{L})$. Other than a thrombocytopenia of $128000 / \mu \mathrm{L}$ there was no abnormality or sign of complicated malaria. Consequently she was treated with mefloquine in a total dose of $1500 \mathrm{mg}$ ( $750 \mathrm{mg}$ followed by 500 $\mathrm{mg} 6 \mathrm{~h}$ later and $250 \mathrm{mg}$ after a further $12 \mathrm{~h}$ ), equivalent to $20 \mathrm{mg} / \mathrm{kg}$ body weight. She did not vomit after taking the drug. Fever had disappeared by day 4 and parasitaemia by day 6 . The patient was discharged on day 7 , clinically fully recovered.

In October 1991 (78 d later) the patient presented again at the same hospital with fever $\left(40^{\circ} \mathrm{C}\right)$, shivering and chills. She had not been abroad since her last hospital admission. Suspecting a $P$. falciparum recrudescence, treatment with halofantrine was started immediately. However, all blood slides sent to us clearly revealed a $P$. malariae monoinfection. She was given 3 doses of halofantrine, $500 \mathrm{mg}$, orally at intervals of $6 \mathrm{~h}$. Again there was no vomiting and recovery was uneventful. Clearance of fever and parasitaemia occurred at days 3 and 4 respectively. Treatment was repeated one week after the initial course, using the same dose of halofantrine.

In January 1992 (106 d after her first admission) the patient presented a third time at the same hospital with fever and headache. Again $P$. malariae was detected in thick and thin blood films sent to us, although she had not travelled to a malaria endemic area since her last admission. Apart from mild splenomegaly no other abnormality could be found and again she was treated with 2 full courses of halofantrine with an interval of one week. Her fever and parasitaemia cleared by days 2 and 8 respectively. After this third treatment the patient was finally referred to us for further counselling and was advised to consult us directly in case of further symptoms. To date (March 1993, $410 \mathrm{~d}$ after her last therapy) she is in good health, no further episode of fever attributable to malarial infection has occurred, and repeated blood films have always been negative for Plasmodium.

In this patient we observed a recrudescence of $P$. malariae infection more than 2 months after mefloquine treatment. There can be no doubt that the drug was sufficiently absorbed, since concomitant infection with $P$. falciparum was cleared promptly. Therefore one might conclude that mefloquine failed to eradicate a very low parasitaemia of $P$. malariae, which had been overlooked at the initial examination. Alternatively it is possible that $P$. malariae parasites were not present in peripheral blood initially, due to a longer incubation period, and, when parasitaemia developed later, the plasma level of mefloquine was no longer sufficient to clear the infection completely.

In the case of halofantrine, on the other hand, poor absorption resulting in low plasma concentrations may have caused the first therapy failure even though a second course was administered after one week, which in falciparum malaria is thought to have almost $100 \%$ efficacy in non-immune subjects (NOTHDURFT et al., 1993). This explanation appears to be more likely than true halofantrine resistance. Such a view is also supported by the fact that the second treatment with halofantrine has obviously resulted in complete cure. Nevertheless, it cannot be ruled out completely that the plasma level of halofantrine needed for effective treatment of $P$. malariae is higher than that for $P$. falciparum.

Interestingly, both clinical episodes of $P$. malariae monoinfection in our patient occurred more than 2 months after the initial mefloquine and halofantrine treatment (78 and $106 \mathrm{~d}$, respectively) and would not have been detected in the usual $28 \mathrm{~d}$ observation period.

We conclude that double infections with $P$. falciparum and $P$. malariae may pose some therapeutic difficulties when treated with mefloquine or halofantrine. Since there has been little experience to date with these 2 drugs in quartan malaria, recrudescence of $P$. malariae after such mixed infections should be treated with chloroquine, which remains the treatment of choice. It is particularly important to make a thorough species diagnosis in such cases before treatment is given.

\section{References}

Cook, G. C. (1988). Prevention and treatment of malaria. Lan cet, ii, 32-36.

Dixon, K. E., Pitaktong, U., Bamnetpandh, S., Teopipithaporn, S. \& Na-Nakorn, A. (1983). Treatment of an acute case of Plasmodium malariae malaria with mefloquine. Fournal of Tropical Medicine and Hygiene, 32, 631-632.

Nothdurft, H. D., Clemens, R., Bock, H. L. \& Löscher, T. (1993). Halofantrine: a new substance for treatment of multidrug-resistant malaria. Clinical Investigator, 71, 69-73.

Weincke, T., Nothdurft, H. D., Kretschmer, H., Fleischer, K., Löscher, T., Braendii, B., Markwalder, K., Schlunk, T., Clemens, R. \& Bock, H. L. 91993). Halofantrine zur Behandlung der importierten Malaria bei nichtimmunen Reisenden. Deutsche Medizinische Wochenschrift, 118, 254-258.

WHO (1992). Expert Committee on Malaria, 19th Report. Geneva: World Health Organization, mimeographed document WHO/CTD/92.1, pp. 9-11.

Received 15 March 1993; accepted for publication 24 March 1993 